\title{
Resource Allocation for Multicarrier CDMA Systems in Ultra-Wideband Communications
}

\author{
Antoine Stephan, Jean-Yves Baudais, and Jean-François Hélard
}

\begin{abstract}
Ultra-wideband (UWB) is a fast emerging technology that has attracted considerable interest in short range, high data rate wireless personal area networks (WPAN) applications. One of the main candidates for WPAN standardization is the multiband orthogonal frequency division multiplexing (MB-OFDM), supported by the Multiband OFDM Alliance (MBOA). In this paper, we propose a new lowcomplexity resource allocation algorithm applied to a spread spectrum multicarrier multiple-access (SS-MC-MA) waveform, which is new for high data rate UWB applications. The proposed scheme aims at maximizing the system's throughput while taking into consideration the WPAN environment and respecting the OFDM parameters of the MBOA solution. The adaptive allocation algorithm applied to OFDM and SS-MC-MA leads to roughly double the throughput compared to the MBOA solution at low attenuation levels. Furthermore, at high attenuation levels, SSMC-MA outperforms the adaptive OFDM. Hence, we conclude that the proposed adaptive SS-MC-MA can especially be advantageously exploited for high attenuation UWB applications.
\end{abstract}

Index Terms-Information theory, multicarrier codedivision multiple-access (MC-CDMA), resource allocation, spread spectrum, ultra-wideband technology, wireless communications.

\section{INTRODUCTION}

$\mathrm{U}$ LTRA-WIDEBAND (UWB) is a fast emerging technology that has attracted considerable interest in the research and standardization communities, due to its ability to provide high data rates at low cost and relatively low power consumption in wireless communication systems. UWB radio has recently been popularized as the future technology for short-range wireless personal area networks (WPAN).

UWB has only been used for military purposes until February 2002 when the Federal Communications

Manuscript received April 14, 2006. This work was supported by the Institute of Electronics and Telecommunications of Rennes (IETR), and by France Télécom R\&D, Rennes, FRANCE, within the contract 46136582.

A. Stephan, Member, IEEE, is a Ph.D. student at the Institute of Electronics and Telecommunications of Rennes (IETR), I.N.S.A., 20 Av. Des Buttes de Coesmes, 35043 Rennes, FRANCE (phone: +33 6 15642872; fax: +33 2 23238439; e-mail: antoine.stephan@ens.insa-rennes.fr).

$\mathrm{J}-\mathrm{Y}$. Baudais, Member, IEEE, is a Researcher at the Institute of Electronics and Telecommunications of Rennes (IETR), I.N.S.A. (e-mail: Jean-Yves.Baudais@insa-rennes.fr).

J-F. Hélard, Senior Member, IEEE, is a Professor at the Institute of Electronics and Telecommunications of Rennes (IETR), I.N.S.A. (e-mail: Jean-Francois.Helard@insa-rennes.fr).
Commission (FCC) agreed on the allocation of $7500 \mathrm{MHz}$ of spectrum for unlicensed use of UWB devices for applications in the 3.1-10.6 GHz frequency band [1]. Since UWB spectrum overlays other existing spectrum allocations and in order to reduce interference with existing services, the FCC's regulations are very stringent and require very low transmitted power. UWB devices must occupy more than $500 \mathrm{MHz}$ of bandwidth in the $3.1-10.6 \mathrm{GHz}$ band and the power spectral density (PSD) must not exceed -41.25 $\mathrm{dBm} / \mathrm{MHz}$ to avoid interference with other services sharing the same bandwidth.

The IEEE 802.15 wireless personal area networks standardization group has organized "task group 3a" to develop a very high data rate physical layer based on UWB signaling [2]. Two main multiple-access techniques have been considered by the task group: a pulse radio transmission using direct-sequence code-division multipleaccess (DS-CDMA) [3], and a multiband orthogonal frequency division multiplexing (MB-OFDM) supported by the Multiband OFDM Alliance (MBOA) [4], [5]. Multiband OFDM modulation format seems to be a very competitive candidate due to its favorable properties to mitigate interference and to achieve high data rate. Other techniques based on a multicarrier code-division multiple-access (MCCDMA) scheme combining OFDM and CDMA have also been proposed in order to improve the UWB signal robustness against narrowband interference and to reach higher data rate [6], [7], [8].

Resource allocation is of major interest in a communication system when we want to optimize its performance. In the UWB environment, the channel response varies slowly in time, and can be considered as quasi-static during one frame. So, we can assume that the channel state information (CSI) is available at the transmitters. Previous resource allocation studies were proposed for the multiband OFDM model [9], [10]. In this paper, we propose an efficient low-complexity power allocation technique for a spread spectrum multicarrier multiple-access (SS-MC-MA) waveform [11], which is new in UWB applications. The SS-MC-MA model improves the system performance and reduces the complexity of the resource allocation. The idea of this allocation is to maximize the total throughput of the system while taking into consideration the WPAN environment and the UWB channel's characteristics and respecting the FCC's regulations.

This paper is organized as follows. In Section II, the multiband OFDM proposal is presented first, followed by a description of the new proposed SS-MC-MA scheme and 
the UWB channel model. Section III details the proposed algorithm for the resource allocation applied to the SS-MCMA scheme. Simulation results showing the interest of the proposed scheme for future high data rate UWB applications are given in Section IV before the conclusion.

\section{SYSTEM MODEL}

\section{A. Multiband OFDM Proposal}

The MBOA approach for UWB systems design is based on the combination of an OFDM modulation with a multibanding technique that divides the $7.5 \mathrm{GHz}$ UWB spectrum into 14 sub-bands of $528 \mathrm{MHz}$ each [12], as shown in Fig. 1. One advantage of multibanding is the ability to process the information over much smaller bandwidth, which reduces power consumption and lowers cost. Initially, most of the studies have been performed on Channel 1 that regroups the first 3 sub-bands (3.1-4.8 $\mathrm{GHz}$ ).

Unique logical channels corresponding to different piconets are defined by using different time-frequency codes (TFC) for each band group. At a given time, each user occupies one of the three sub-bands of Channel 1 . The time frequency codes provide frequency hopping from a subband to another at the end of each OFDM symbol, which allows every user to benefit from the frequency diversity related to a bandwidth equal to 3 sub-bands.

The MBOA system is capable of transmitting data at information rates going from 53.3 to $480 \mathrm{Mb} / \mathrm{s}$. The OFDM scheme consists of 128 subcarriers. Out of the 128 subcarriers, only 122 tones carry energy. From these used tones, 100 are assigned to data tones, 12 to pilot tones, and 10 to guard tones. The 122 subcarriers are modulated using quadrature phase-shift keying (QPSK). The useful duration of each OFDM symbol is $242 \mathrm{~ns}$, leading to a subcarrier frequency spacing of $4.125 \mathrm{MHz}$. A zero-padding cyclic prefix of duration 60.61 ns is added at the end of each OFDM symbol to cope with the inter-symbol interference (ISI). An additional guard interval of 9.47 ns is added for the sub-bands hops. The main OFDM parameters of the MBOA solution are given in Table I.

In brief, the MBOA solution offers potential advantages for high data rate UWB applications, like for example the signal robustness against channel selectivity and the efficient exploitation of the energy of every signal received within the prefix margin. However, the MBOA solution has some limitations in a multi-user and multi-piconet context. For example, if we consider only the first 3 sub-bands of Channel 1, conflicts between users start as soon as we add a fourth user to the cell, whereas cells of up to 6 simultaneous users are being considered. Besides, this solution lacks in the ability to allocate sub-bands and power optimally since the sub-bands are not assigned to each user according to his channel's condition, and the transmit power is equally distributed among sub-bands without any power adaptation.

\section{B. $M C-C D M A$}

Some studies have proposed to add a CDMA scheme to the MBOA solution by spreading user's signals in the

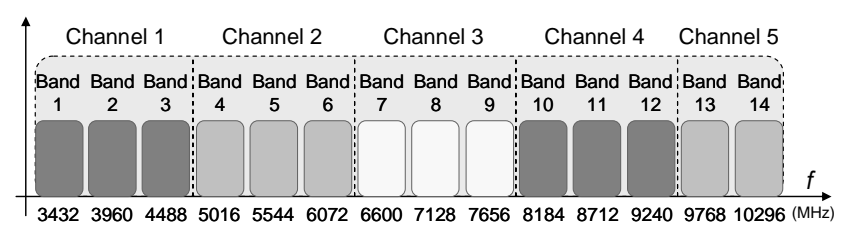

Fig. 1. Channels and sub-bands distribution for MBOA.

TABLE I

OFDM PARAMETERS OF MBOA SOLUTION

\begin{tabular}{lc}
\hline \hline \multicolumn{1}{c}{ Parameter } & Value \\
\hline FFT size & 128 \\
Sampling frequency & $528 \mathrm{MHz}$ \\
Transmission band & $507.37 \mathrm{MHz}$ \\
Number of data subcarriers & 100 \\
Number of pilot carriers & 12 \\
Number of guard carriers & 10 \\
Total number of subcarriers used & 122 \\
Subcarrier frequency spacing & $4.125 \mathrm{MHz}$ \\
IFFT/FFT period & $242.42 \mathrm{~ns}$ \\
Zero pad duration & $70.08 \mathrm{~ns}$ \\
Symbol interval & $312.5 \mathrm{~ns}$ \\
\hline \hline
\end{tabular}

frequency domain, in order to improve the system performance [6], [7], [8]. The resource allocation becomes more flexible with MC-CDMA due to the addition of a spreading sequence. In fact, a tradeoff between overall transmission rate and quality of service from frequency diversity is allowed. In addition, the spreading in the frequency domain improves the signal robustness against the frequency selectivity of the UWB channel, and against the narrowband interference, since the signal bandwidth could become much wider than the interference's bandwidth. However, some authors suggest using a MCCDMA with a bandwidth of $1.58 \mathrm{GHz}$ corresponding to 3 sub-bands of a MBOA signal [13], which leads to highly increase the required sampling frequency of the analog-todigital conversion.

\section{Proposed scheme: SS-MC-MA}

In order to improve the resource allocation more than in the MC-CDMA case, we propose a SS-MC-MA scheme. SS-MC-MA can be considered as a MC-CDMA technique that introduces an additional frequency division multiple access (FDMA) at the subcarrier level to avoid multiple access interference (MAI) [14]. The main difference between SS-MC-MA and MC-CDMA is that with SS-MCMA the code division is used for the simultaneous transmission of the data symbols of a single user on a given subset of subcarriers, while with MC-CDMA, it is used for the simultaneous transmission of different users' symbols. SS-MC-MA assigns each user exclusively its own subset of subcarriers according to the FDMA scheme.

The SS-MC-MA system benefits from the performance and advantages of the MC-CDMA while improving the resource allocation flexibility. Another advantage over MCCDMA scheme is that the SS-MC-MA channel estimation at the reception is less complex. In fact, each subcarrier is only affected by one user's channel, whereas in a MC-CDMA system each subcarrier is affected by the different channels 


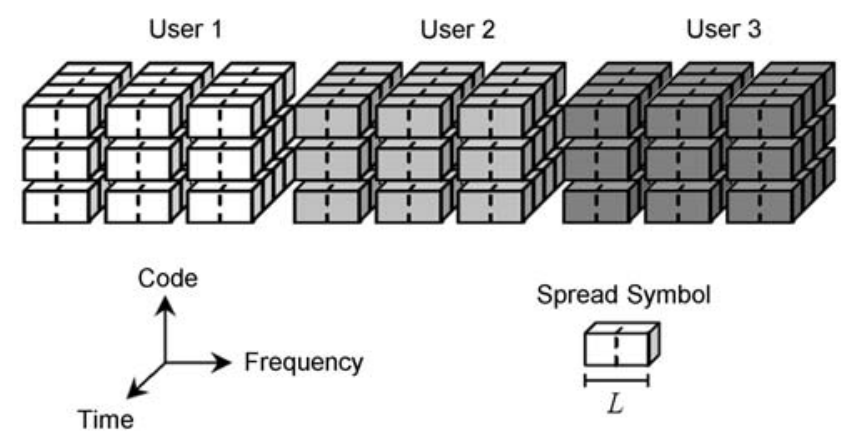

Fig. 2. SS-MC-MA system architecture.

of the different users.

The SS-MC-MA system used in this paper for the resource allocation is represented in Fig. 2. At a given time, each user is allocated a group of subcarriers equivalent to one of the first $3 \mathrm{MBOA}$ bands of $528 \mathrm{MHz}$ bandwidth each. Then, each band is divided into several sub-bands of bandwidth equal to the spreading code length $L$. However, as we will see later on, the subcarriers linked within a given sub-band by the $C$ spreading codes of a given user are not necessarily adjacent. Besides, the OFDM parameters of the MBOA solution, presented in Table I, are maintained.

The constellation used in the MBOA solution is a QPSK. With the proposed SS-MC-MA model, we also use 8-QAM (quadrature amplitude modulation) and 16-QAM constellations in order to make the allocation more flexible and to better fit the channel capacity. The maximum number of bits per constellation is limited to 4 , which corresponds to the case of 16-QAM, in order not to increase very much the number of quantization bits of the analog-to-digital conversion.

\section{Channel Model}

The channel model used for our resource allocation is the one adopted by the IEEE 802.15a committee for the evaluation of UWB physical layer proposals [15]. It is a modified version of the Saleh-Valenzuela model for indoor channels [16] that fits the properties of measured UWB channels. A lognormal distribution is used for the multipath gain magnitude. In addition, independent fading is assumed for each cluster and each ray within the cluster.

The impulse response of the multipath model is described as

$$
h_{k}(t)=X_{k} \sum_{m=0}^{M_{k}} \sum_{p=0}^{P_{k}} \alpha_{k}(m, p) \delta\left(t-T_{k}(m)-\tau_{k}(m, p)\right) \text {, }
$$

where $X_{k}$ is the log-normal shadowing, $T_{k}(m)$ the delay of

TABLE II

MULTIPATH CHANNEL CHARACTERISTICS

\begin{tabular}{lllll}
\hline \hline & CM1 & CM2 & CM3 & CM4 \\
\hline Mean excess delay & 5.05 & 10.38 & 14.18 & \\
Delay spread & 5.28 & 8.03 & 14.28 & 25 \\
Distance & $<4$ & $<4$ & $4-10$ & 10 \\
LOS/NLOS & LOS & NLOS & NLOS & NLOS \\
\hline \hline
\end{tabular}

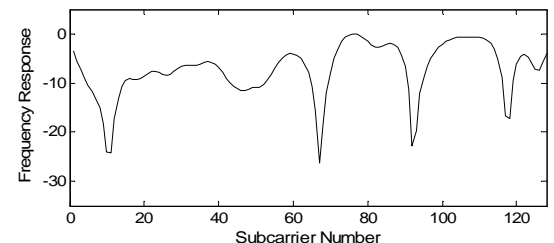

(a) Frequency response of CM1 model.

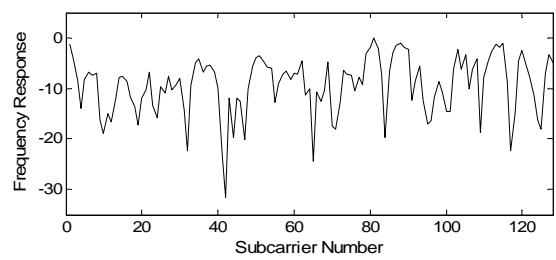

(b) Frequency response of CM4 model.

Fig. 3. A typical realization of UWB channel models CM1 and CM4.

the $m^{\text {th }}$ cluster, $\alpha_{k}(m, p)$ and $\tau_{k}(m, p)$ represent the gain and the delay of the $p^{\text {th }}$ multipath within the $m^{\text {th }}$ cluster respectively.

Four different channel models (CM1 to CM4) are defined for the UWB system modeling, each with arrival rates and decay factors chosen to match different usage scenarios and to fit line-of-sight (LOS) and non-line-of-sight (NLOS) cases. The main characteristics of the different channel models are given in Table II, and a typical realization of the frequency responses of CM1 and CM4 models is represented in Fig. 3. As expected from Table II, we can see that the channel frequency diversity of CM4 model is much higher than the one of CM1 model.

\section{PRoposed AlgorithM}

Previous resource allocation algorithms were proposed for the multiband OFDM model [9], [10]. Here, we propose an efficient algorithm for the SS-MC-MA waveform described above, in a UWB environment. This algorithm will study the bit rate maximization problem (BRMP) case [17], i.e., it will maximize the total throughput with a PSD constraint fixed by the FCC's regulations. It is assumed that the channel state information is available at the transmitters.

Using Shannon's theorem, the capacity of an OFDM system can be written as

$$
C_{\text {OFDM }}=\sum_{k \in S} \log _{2}\left(1+\left|H_{k}\right|^{2} \frac{E_{k}}{N_{0}}\right),
$$

where $S$ is the group of used subcarriers, $H_{k}$ and $E_{k}$ the frequency-domain response and the transmitted power density of the $k^{\text {th }}$ subcarrier respectively, and $N_{0}$ the noise density.

Now, we consider a system composed of a single SS-MCMA block for a single user. The system throughput per symbol for a single SS-MC-MA block using a zero-forcing detection is given from [18] by

$$
R_{S S-M C-M A}=\sum_{c=0}^{C-1} \log _{2}\left(1+\frac{1}{\Gamma} \frac{L^{2}}{\sum_{k \in S} \frac{1}{\left|H_{k}\right|^{2}}} \frac{E_{c}}{N_{0}}\right),
$$


where $C$ is the number of codes, $S$ the group of subcarriers, $L$ the spreading code length, $H_{k}$ the frequency-domain response of the $k^{\text {th }}$ subcarrier, $E_{c}$ the power density of the $c^{\text {th }}$ code, and $\Gamma$ the signal-to-noise ratio (SNR) gap, known as the normalized SNR [19].

The throughput maximization study is divided into two parts. In the first part, we consider an infinite granularity case where the throughput $R$ can have real non-integer values, and in the second part we consider a finite granularity case where the throughput values must be integer as we have discrete modulation orders.

\section{A. Infinite Granularity}

In the infinite granularity case, by imposing a constraint on the PSD, the optimization problem can be described as

$$
\max \sum_{c=0}^{C-1} \log _{2}\left(1+\frac{1}{\Gamma} \frac{L^{2}}{\sum_{k \in S} \frac{1}{\left|H_{k}\right|^{2}}} \frac{E_{c}}{N_{0}}\right) \text {, }
$$

subject to

$$
\sum_{c=0}^{C-1} E_{c} \leq E
$$

where $E$ is the PSD constraint.

To maximize the system throughput for a given PSD, the $L$ subcarriers that have the largest response amplitude $\left|H_{k}\right|$ should be selected. Let $S^{*}$ be the group of the $L$ subcarriers with the largest amplitude.

To solve the optimization problem, we introduce the Lagrange multipliers $\lambda$ in (4) and (5). We find that

$$
\begin{aligned}
E_{c} & =\frac{E}{C}, \\
C & =L .
\end{aligned}
$$

Then, the maximum reachable throughput $R^{*}$ of the whole system in the infinite granularity case becomes

$$
R^{*}=L \log _{2}\left(1+\frac{1}{\Gamma} \frac{L}{\sum_{k \in S^{*}} \frac{1}{\left|H_{k}\right|^{2}}} \frac{E}{N_{0}}\right),
$$

and the maximum reachable throughput per code $c$ becomes

$$
R_{c}^{*}=\frac{R^{*}}{L} \quad \forall \mathrm{c} \in[0: \mathrm{L}-1]
$$

\section{B. Finite Granularity}

As we have discrete modulation orders, the throughput per code should have integer values. One solution is to allocate for each code a number of bits equal to the floor of the maximum throughput per code found in (9),

$$
\bar{R}_{c}=\left\lfloor R^{*} / L\right\rfloor,
$$

where $\bar{R}_{c}$ is the discrete throughput of the $c^{\text {th }}$ code. It is clear that this solution respects the PSD constraint since the total throughput is lower than the system capacity. Also, we notice that we might be able to increase the throughput of some codes while respecting the PSD constraint.

An optimal solution for this problem is given in [18]. It proves that an optimal throughput distribution would be to allocate $\left\lfloor R^{*} / L\right\rfloor+1$ bits to $n$ codes and $\left\lfloor R^{*} / L\right\rfloor$ bits to the remaining $L-n$ codes, where $n$ is an integer number given by

$$
n=\left\lfloor L\left(2^{R^{*} / L-\left\lfloor R^{*} / L\right\rfloor}-1\right)\right\rfloor .
$$

From the optimal throughput distribution, $\bar{R}_{c}$ can be expressed as

$$
\begin{cases}\bar{R}_{c}=\left\lfloor R^{*} / L\right\rfloor+1 & \forall c \in[0: n-1] \\ \bar{R}_{c}=\left\lfloor R^{*} / L\right\rfloor & \forall c \in[n: L-1]\end{cases}
$$

The power density $\bar{E}_{c}$ of the $c^{\text {th }}$ code can be computed using $\bar{R}_{c}$ value obtained from (12),

$$
\bar{E}_{c}=\frac{\Gamma}{L^{2}} \sum_{k \in S^{*}} \frac{N_{0}}{\left|H_{k}\right|^{2}}\left(2^{\bar{R}_{c}}-1\right) .
$$

Finally, the total discrete system throughput $\bar{R}$ is given by

$$
\begin{aligned}
\bar{R}=\left(\left\lfloor L\left(2^{R^{*} / L-\left\lfloor R^{*} / L\right\rfloor}-1\right)\right\rfloor\right)\left(\left\lfloor R^{*} / L\right\rfloor+1\right) \\
+\left(L-\left\lfloor L\left(2^{R^{*} / L-\left\lfloor R^{*} / L\right\rfloor}-1\right)\right\rfloor\right)\left(\left\lfloor R^{*} / L\right\rfloor\right) .
\end{aligned}
$$

\section{Implementation}

After having presented the proposed algorithm for a single given SS-MC-MA block of $L$ subcarriers, the bit loading algorithm is now applied to the whole MBOA subbands of $N=100$ used subcarriers each. The total number of blocks per sub-band is equal to $\lfloor N / L\rfloor$.

This algorithm can be implemented as follows.

1) Initialization

Set $R_{\text {code, block }}=0$ and $E_{\text {code, block }}=0, \forall$ code, block,

$S_{\text {block }}=\phi, \forall$ block,

$L=$ spreading code length,

$N=$ number of data subcarriers,

$H=\left\{H_{1}, \ldots, H_{N}\right\}$.

2) For block $=1$ to $\lfloor N / L\rfloor$,

find $S_{\text {block }}=$ best $L$ subcarriers in $H$,

update $H$,

$$
H=H-S_{\text {block }},
$$

find the system throughput $R^{*}$ from (8), compute the code throughput $R_{\text {code, block }}$, 


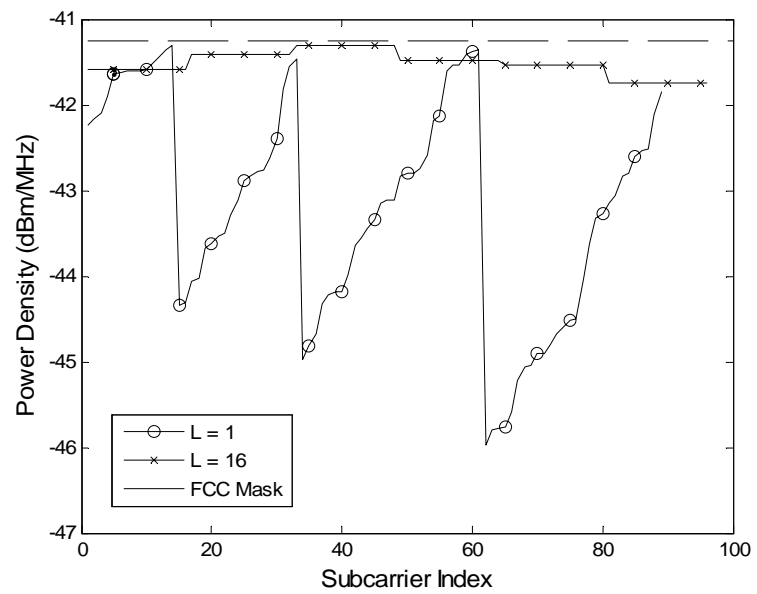

Fig. 4. Power spectral density of the transmitted signal.

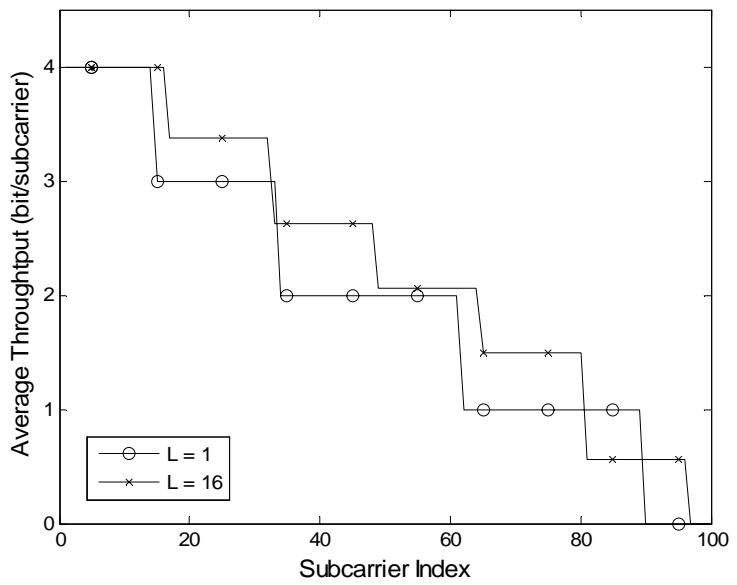

Fig. 5. Average throughput per used subcarrier.

$$
\begin{aligned}
& \text { for code }=0 \text { to } n-1, \\
& R_{\text {code }, \text { block }}=\left\lfloor R^{*} / L\right\rfloor+1, \\
& \text { for code }=n \text { to } L-1, \\
& R_{\text {code }, \text { block }}=\left\lfloor R^{*} / L\right\rfloor,
\end{aligned}
$$

where $n$ is given by (11),

compute the code power density $E_{\text {code, block }}$,

$$
E_{\text {code }, \text { block }}=\frac{\Gamma}{L^{2}} \sum_{k \in S_{\text {block }}} \frac{N_{0}}{\left|H_{k}\right|^{2}}\left(2^{R_{\text {code }, \text { block }}}-1\right) .
$$

3) Output

$$
\text { Return } S_{\text {block }}, R_{\text {code, block }} \text { and } E_{\text {code, block }} \text {. }
$$

\section{Simulation RESUlTS}

In this section, we present the results of the simulations performed on the first sub-band of the multiband OFDM solution for the channel models CM1 and CM4, using the proposed adaptive SS-MC-MA system.

Fig. 4 and Fig. 5 represent the power spectral density and the average throughput per used subcarrier respectively, for 2 different spreading code lengths $L=1$ and $L=16$. The code length $L=1$ corresponds to a classical OFDM system with adaptive modulation, while $L=16$ is the optimal code length of SS-MC-MA, that gives the highest system

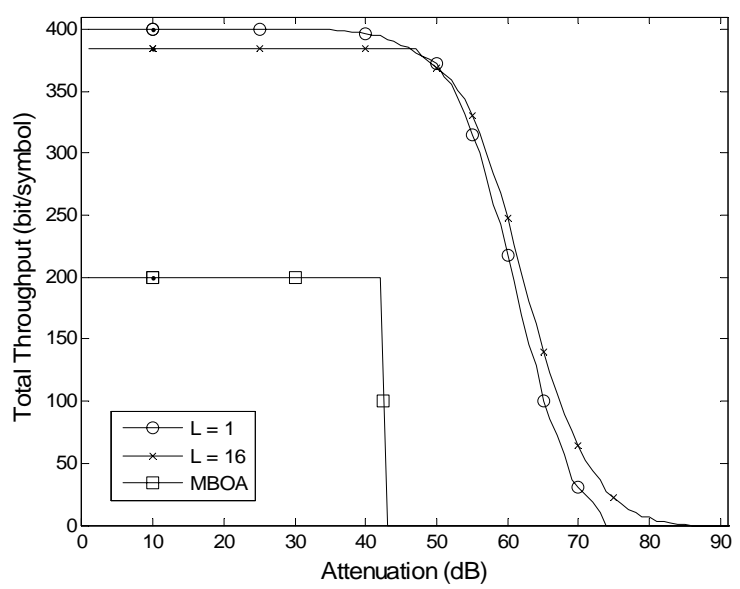

Fig. 6. Total throughput per OFDM symbol for different attenuation levels with the CM1 channel model.

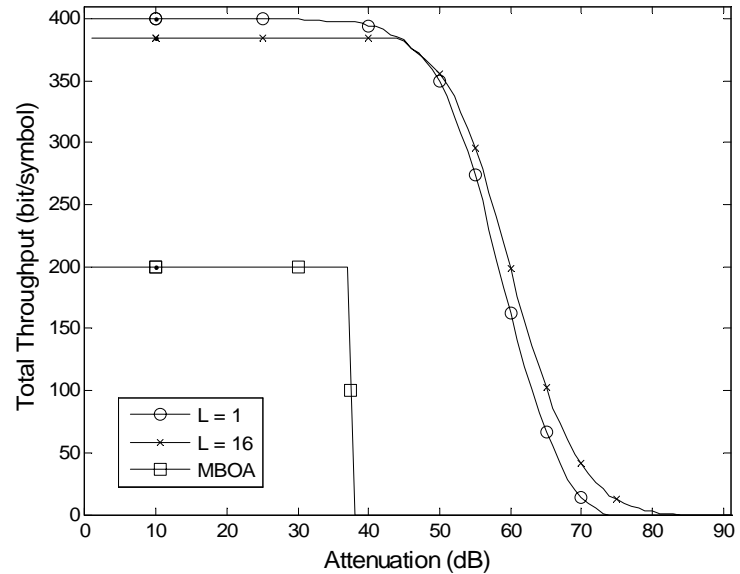

Fig. 7. Total throughput per OFDM symbol for different attenuation levels with the CM4 channel model.

throughput even if only 96 subcarriers can be used in this case ( 6 blocks of 16 subcarriers each). The spreading codes are orthogonal and extracted from the Hadamard's matrices of size $L \times L$. From Fig. 4, we can notice that with $L=16$, the system benefits from the available PSD much more than with $L=1$; the PSD of the transmitted signal with $L=16$ is almost flat and very close to the FCC's limit of -41.25 $\mathrm{dBm} / \mathrm{MHz}$. This is due to the fact that with $L=16$ the average number of bits per subcarrier is not necessarily integer anymore, as we can see in Fig. 5.

Fig. 6 and Fig. 7 represent the total throughput per OFDM symbol for different channel attenuation levels, with the CM1 and CM4 channel models respectively. These attenuations represent the link loss of the channel. For an attenuation of $0 \mathrm{~dB}$, the maximum amplitude of the frequency channel coefficients is $\left|H_{k}\right|=1$. For an attenuation of around $70 \mathrm{~dB}$, the PSD of the received signal is lower than the noise density $N_{0}=-114 \mathrm{dBm} / \mathrm{MHz}$.

The system throughput is evaluated without channel coding and for a target symbol error rate of $10^{-3}$. For example, in Fig. 6 and Fig. 7, the throughput of 200 bit/symbol with the MBOA solution corresponds to the total useful bit rate of $320 \mathrm{Mbps}$ transmitted in a $528 \mathrm{MHz}$ band with the typical MBOA mode, using a convolutional coding 
rate of $1 / 2$. We see that this $200 \mathrm{bit} / \mathrm{symbol}$ throughput can only be reached for attenuations lower than around $40 \mathrm{~dB}$ with MBOA. At any attenuation level, the adaptive SS-MCMA ( $L=16$ ) using higher modulation orders (8-QAM and 16-QAM), as well as the adaptive OFDM $(L=1)$, outperforms MBOA. The proposed adaptive allocation applied to OFDM or SS-MC-MA leads to roughly double the throughput at a $40 \mathrm{~dB}$ attenuation level for CM1 and CM4 channel models. The total useful bit rate is slightly higher with adaptive OFDM (400 Mbps) than with adaptive SS-MC-MA (384 Mbps) for attenuation levels lower than $40 \mathrm{~dB}$. This is due to the number of used data subcarriers which is equal to 100 with OFDM and 96 with SS-MC-MA.

However, for attenuation levels higher than $50 \mathrm{~dB}$, adaptive SS-MC-MA $(L=16)$ is better than adaptive OFDM ( $L=1)$, and of course outperforms MBOA. The adaptive allocation for SS-MC-MA and OFDM schemes provides the same MBOA throughput of $200 \mathrm{bit} / \mathrm{symbol}$ at attenuation levels of $63 \mathrm{~dB}$ and $60 \mathrm{~dB}$ respectively, with CM1 channel model. The reason is that the adaptive OFDM scheme is not able to exploit the total amount of energy available on each subcarrier in the case of PSD constraint with finite modulation orders, while the spreading component of SS-MC-MA allows the subcarriers of a same sub-band to pool their energy to transmit one or more additional bits.

This adaptive system can also transmit information at an attenuation level of $73 \mathrm{~dB}$ with OFDM, and $85 \mathrm{~dB}$ with SSMC-MA. We should notice that the system is able to transmit information even when the PSD is lower than the noise floor. This is due to the spreading gain that is equal to $10 \log _{10}(L)=12 \mathrm{~dB}$ with $L=16$.

Other simulations have also been performed on the second and third sub-bands of the multiband OFDM scheme for the channel models CM1, CM2, CM3 and CM4. Similar results are obtained, highlighting the throughput gain of the proposed adaptive SS-MC-MA scheme compared to the MBOA solution.

\section{CONCLUSION}

In this paper, we proposed a new low-complexity resource allocation algorithm applied to a SS-MC-MA waveform for high data rate UWB applications. The proposed scheme aims at maximizing the system's throughput while taking into consideration the WPAN environment and respecting the FCC's regulations. The new SS-MC-MA waveform also respects the OFDM parameters of the MBOA solution. The adaptive allocation algorithm applied to OFDM and SS-MC-MA leads to roughly double the throughput compared to the MBOA solution at low attenuation levels for any channel model. Furthermore, at high attenuation levels, SS-MC-MA outperforms adaptive OFDM. This is due to the energy gathering capability of SSMC-MA within each sub-band, which can exploit, contrary to OFDM, the residual energy conveyed by each subcarrier. Hence, we conclude that the proposed adaptive SS-MC-MA can especially be advantageously exploited for high attenuation UWB applications.

\section{REFERENCES}

[1] "First report and order, revision of part 15 of the commission's rules regarding ultra-wideband transmission systems,” FCC, ET Docket 98153, Feb. 14, 2002.

[2] IEEE 802.15 WPAN High Rate Alternative PHY Task Group 3a (TG3a). Internet: www.ieee802.org/15/pub/TG3a.html.

[3] Q. Li and L. Rusch, "Multiuser detection for DS-CDMA UWB in the home environment," IEEE J. on Selected Areas in Commun., vol. 20, no. 9, pp. 1701-1711, Dec. 2002.

[4] IEEE P802.15 Working Group for Wireless Personal Area Networks (WPANs), "Multi-band OFDM physical layer proposal for IEEE 802.15 task group 3a,” Sept. 2004.

[5] A. Batra et al., "Design of multiband OFDM system for realistic UWB channel environments," IEEE Trans. on Microwave Theory and Techniques, vol. 52, no. 9, Sept. 2004.

[6] M. Schmidt and F. Jondral, "Ultra wideband transmission based on MC-CDMA,” IEEE GLOBECOM Conference, 2003.

[7] W. Tung and J. Wang, "MMSE receiver for multicarrier CDMA overlay in ultra-wide-band communications," IEEE Trans. on Vehicular Technology, vol. 54, no. 2, pp. 603-614, March 2005.

[8] J. Wang and L. Milstein, "Multicarrier CDMA overlay for ultrawideband communications,” IEEE Trans. Commun., vol. 52, no. 10, pp. 1664-1669, Oct. 2004.

[9] Z. Xu and L. Liu, "Power allocation for multi-band OFDM UWB communication networks," IEEE Vehicular Technology Conference, vol. 1, pp. 368-372, Sept. 2004.

[10] W. Siriwongpairat, Z. Han and K. Ray Liu, "Energy-efficient resource allocation for multiband UWB communication systems," Wireless Communications and Networking Conference, vol. 2, pp. 813-818, March 2005

[11] L. Cariou and J-F. Hélard, “A novel uplink transmission system based on MIMO frequency hopping SS-MC-MA for the future wireless cellular networks," Telecommunications System Journal, vol. 30, no. 1-3, pp. 193-214, Nov. 2005.

[12] A. Batra et al., "TI physical layer proposal for IEEE 802.15 task group 3a,” IEEE P802.15-03/142r2-TG3a, Mar. 2003.

[13] Y-B. Park et al., "Performance of UWB DS-CDMA/OFDM/MCCDMA systems," $47^{\text {th }}$ IEEE International Midwest Symposium on Circuit and Systems, 2004.

[14] S. Kaiser and K. Fazel, "A flexible spread-spectrum multi-carrier multiple-access system for multi-media applications,” in Proc. IEEE Intern. Symp. on Personal, Indoor and Mobile Radio Commun. (PIMRC'97), pp. 100-104, Helsinki, Finland, Sept. 1997.

[15] J. Foerster et al., "Channel modeling sub-committee report final," IEEE802.15-02/490, Nov. 2003.

[16] A. Saleh and R. Valenzuela, "A statistical model for indoor multipath propagation,” IEEE J. on Selected Areas in Commun., vol. 5, no. 2, pp. 128-137, Feb. 1987.

[17] J. Campello, "Practical bit loading for DMT," in IEEE International Conference on Communications, pages 801-805, 1999.

[18] M. Crussière, J-Y. Baudais and J-F. Hélard, “Adaptive spread spectrum multicarrier multiple access over wirelines,” IEEE $J$. on Selected Areas in Commun., Power Line Communication, July 2006, to be published.

[19] J.M. Cioffi, “A multicarrier primer,” Rapport, ANSI T1E1.4/91-157, Committee contribution, Tech. Rep., 1991. 